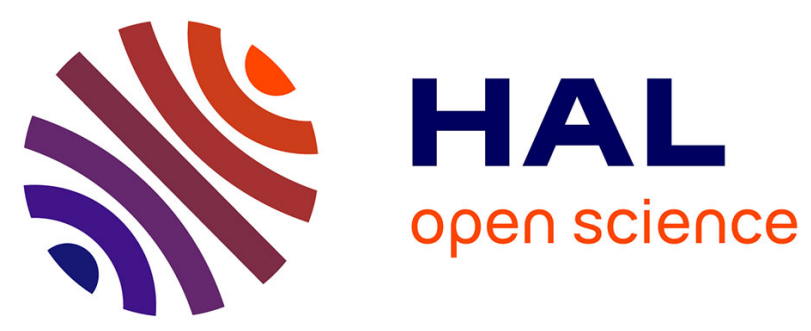

\title{
New method for decoupling the articular stiffness identification: Application to an industrial robot with double encoding system on its 3 first axis
}

\author{
Alexandre Ambiehl, Sébastien Garnier, Kévin Subrin, Benoit Furet
}

\section{- To cite this version:}

Alexandre Ambiehl, Sébastien Garnier, Kévin Subrin, Benoit Furet. New method for decoupling the articular stiffness identification: Application to an industrial robot with double encoding system on its 3 first axis. IEEE/RSJ International Conference on Intelligent Robots and Systems, Sep 2017, Vancouver, Canada. 10.1109/IROS.2017.8205950 . hal-01693066

\section{HAL Id: hal-01693066 https://hal.science/hal-01693066}

Submitted on 22 Oct 2018

HAL is a multi-disciplinary open access archive for the deposit and dissemination of scientific research documents, whether they are published or not. The documents may come from teaching and research institutions in France or abroad, or from public or private research centers.
L'archive ouverte pluridisciplinaire HAL, est destinée au dépôt et à la diffusion de documents scientifiques de niveau recherche, publiés ou non, émanant des établissements d'enseignement et de recherche français ou étrangers, des laboratoires publics ou privés. 


\title{
New method for decoupling the articular stiffness identification : application to an industrial robot with double encoding system on its 3 first axis
}

\author{
Alexandre Ambiehl ${ }^{1}$, Sébastien Garnier ${ }^{2}$, Kévin Subrin ${ }^{2}$ and Benoit Furet ${ }^{2}$
}

\begin{abstract}
In order to be able to perform complex and arduous tasks, stiffness articular identification of industrial robots is a current approach to predict the deflection under static or dynamic loading. Manufacturers propose new features to take the loading into account and a new generation of industrial robot equiped with double encoding systems are proposed. However, current methods brings some drawbacks when the ratio between the stiffness arm and the wrist one is too high. In this paper, we propose a new approach to take this aspect into account by decoupling the arm identification and the wrist one. We compare then our method regarding two current methods and applied it on this new industrial robot. The results highligh the stability and the quality of the stiffness articular estimation with and without activating the double encoding system. On our data, we are able to take into account $84 \%$ of the global deflection.
\end{abstract}

\section{INTRODUCTION}

The increasing competitiveness between companies requires means of production which meet an expected functionality at a reduced investment cost. This financial rationalization encourages industrial robotic to compete with machine tools as regards tasks for which the volume of the workspace is large and does not require a great accuracy (up to $0.1 \mathrm{~mm}$ ). However, the behavior of robots must be improved to answer more specifically to the needs of customers in terms of accuracy and quality. Manufacturers propose new and adapted manipulators architectures. These improvements are represented through the integration of more rigid gearboxes, more powerful engines, mass compensator, parallelogram closed loop, the integration of the spindle in the $5^{\text {th }}$ link. This paper focuses on a new feature i.e. the integration of a double encoding systems for a better control of the 3 first axis during strong loadings. In fact, the main deflection on a serial robot is localized at its joints [1]. This integration allows to delete a part of the deflection inside the axis (axes torsion, elastic deformation of the reductors). In the rest of the paper, we will consider as "arm" the 3 first axis and we call "wrist" the 3 last one of the robot.

Currently, many work proposes ways to improve the accuracy and more generally the behavior of industrial robot. In fact, due to its serial architecture, the robot has an

\footnotetext{
${ }^{1}$ Alexandre Ambiehl, Gebe2productique, Europetechnologies group, Parc activité Vendée Sud loire, 85600 Boufféré, France a.ambiehl@europetechnologies.com

${ }^{2}$ S. Garnier, K. Subrin and F. Furet are with ROMAS Team, LS2N Research Laboratory University of Nantes, IUT, 2 av Prof Jean Rouxel 44470 Carquefou, France - \{sebastien.garnier;kevin.subrin;benoit.furet\}@univnantes.fr
}

anisotropic behavior in its workspace i.e. its performance is dependent on its positioning, its configuration, its distance to its singularity. Then, works rely on the identification of the whole parameters of an industrial robot (link length, offset, kinematic parameters, dynamic parameters) to improve its absolute accuracy and its behavior regarding the task it must perform. Many factors influence the accuracy of the robot such as elastic deformation[2], [3], thermal deformation[4], modelling errors[5], [6] . Identification is performed via online or offline methods [7], [8]. As far as stiffness identification is concerned, main approaches are offline based on an exteroceptive or proprioceptive measurement of the deflection of the robot due to a static weight. Current approaches on this subject have some limits we will discuss and we present here a new method that we compare with what authors proposed [9], [10].

This paper is organized as follows. Section II presents the stiffness modelling background. Section III defines the new method based on a decoupling approach to first identify the 3 first axis and the 3 last one. Section IV presents the results via the use of 3 methods on the industrial robot with double encoding system. Section $\mathrm{V}$ gives the conclusion and the perspective of the paper.

\section{STIFFNESS MODELLING BACKGROUND}

Conservation Congruence Transformation(CCT) was proposed by Chen and Kao [1] to define the spatial Cartesian stiffness matrix of a serial robot. We first define the notation and we develop the approach.

\section{A. Jacobian Matrix}

The Jacobian matrix ${ }^{p} J_{h}$ of the robot relates the joint rates $\dot{q}$ to the twist of the end effector namely.

$$
\left[\begin{array}{l}
{ }^{p} V \\
{ }^{p} w
\end{array}\right]=\left[{ }^{p} J_{h}\right][\dot{q}]
$$

${ }^{p} V$ is the translation speed of the end effector expressed in $p$ coordinate system, ${ }^{p} w$ is the rotation speed of the end effector expressed in $p$ coordinate system, $\dot{q}$ is the articular speed, $\left[{ }^{p} J_{h}\right]$ is the Jacobian matrix which defines the speed of $h$ element expressed in $p$ coordinate system.

The static model of the robot can be introduced in the same way.

$$
\Gamma=\left[{ }^{p} J_{h}\right]^{T}\left[{ }^{p} W_{h}\right]
$$


with $\Gamma$ the torque applied to the joint, ${ }^{p} J_{h}$ the Jacobian matrix and ${ }^{p} W_{h}$ the wrench applied to the end effector.

\section{B. Cartesian stiffness matrix formulation via complete pose method}

The cartesian stiffness matrix of a robot depends on its configuration, links stiffness, control loop stiffness and actuators mechanical stiffness. In this paper, the last two sources of stiffness are considered. The links of the robot are assumed to be rigid, the damping is neglected and the stiffness of the joints is represented with linear torsional springs. The spatial cartesian stiffness of a serial robot can be defined by:

$$
W=K_{x} \cdot \Delta X
$$

with

$$
K_{x}=J^{-T} \cdot\left(K_{\theta}-K_{c}\right) J^{-1}
$$

$W$ is the six-dimensional wrench vector composed of the forces and torques applied on the end-effector on a given point and expressed in $R_{0} . K_{X}$ is the $6 \times 6$ cartesian stiffness matrix of the robot expressed in $R_{0} . \Delta X$ is the six-dimensional vector composed of the translational and rotational displacements of the end-effector expressed in $R_{0}$. $J$ is the Jacobian matrix of the robot defined in (1). $K_{\theta}$ is the diagonal joint stiffness matrix defined as follows:

$$
K_{\theta}=\left(\begin{array}{cccccc}
k_{\theta_{1}} & 0 & \ldots & \ldots & \ldots & 0 \\
0 & k_{\theta_{2}} & \ddots & & & \vdots \\
\vdots & \ddots & k_{\theta_{3}} & & \ddots & \vdots \\
\vdots & & \ddots & k_{\theta_{4}} & \ddots & \vdots \\
\vdots & & & \ddots & k_{\theta_{5}} & 0 \\
0 & \ldots & \ldots & \ldots & 0 & k_{\theta_{6}}
\end{array}\right)
$$

Dumas et al. [9] highlight that to facilitate the estimation of the stiffness identification that $K_{c}$ must be negligle. They present the definition of a criteria to have a good conditioning of the Jacobian matrix and they place the robot in such a way that, they can lead to the following approximation :

$$
K_{x} \approx J^{-T} K_{\theta} J^{-1}
$$

The stiffness identification takes the form :

$$
W=J^{-T} K_{\theta} J^{-1} \delta p \quad \text { with } \quad \delta p=\left[\begin{array}{ll}
\delta p_{m} & \delta p_{\text {rad }}
\end{array}\right]^{T}
$$

For a given configuration, the measurement of the deflection $(\delta p)$ in translation $\left(\delta p_{m}\right)$ and rotation $\left(\delta p_{\text {rad }}\right)$ due to a wrench $W$ allows to identify the stiffness via the Complete Pose method. Unfortunately, this equation introduces various bottlenecks. On one hand, we observe some compensation effects. The stiffness values for the 3 first axes of the robot and the 3 last one have a ratio of 10 (See Tab. II). During the identification, we can observe a over-estimation of the 3 first axis leading to a negative stiffness. That has no physical meaning. On the other hand, the position and the orientation with and without loading is measured. However, it is not homogeneous (Length in meter and orientation in rad). To take this aspect into account, a common approach concerns the normalization of the Jacobian Matrix by means of a normalizing length called characteristic length and denoted as L [11]. Unfortunately, this value depends on a numerical optimization, it is not single and depends on the initial condition to be determined. This normalization will be used in section IV. Lets $J_{N}$ to be the normalized Jacobian matrix

$$
J_{N}=\left[\begin{array}{cc}
\frac{1}{L} I_{3 X 3} & 0_{3 X 3} \\
0_{3 X 3} & I_{3 X 3}
\end{array}\right]
$$

In order to avoid the normalization, an approach leading by[10], [12] allows an identification with partial pose which takes into account only the position of the robot $\delta p_{m}$.

\section{Decoupling the measurement of the displacement point and the force application point}

Equation (7) lets appear the same Jacobian and it is necessary, for the identification, to define at the same point the loading and the displacement. Unfortunately, it implies to have a deep knowledge of the geometry which is not perfect. Based on that, we can introduce in the formula, the Jacobian for the loading $J_{w}$ and the Jacobian for the displacement $J_{m}$. In fact, we can write :

$$
\Gamma=J_{w}^{T} f \quad \delta p=J_{m} \delta \theta \quad \Gamma=K_{\theta} \delta \theta \quad f=K_{x} \delta p
$$

Taking the partial derivatives of (17) and

$$
\frac{\partial \Gamma}{\partial \theta}=\frac{\partial J_{w}^{T}}{\partial \theta} f+J_{w}^{T} \frac{\partial f}{\partial p} \frac{\partial p}{\partial \theta}
$$

neglecting $K_{c}=\frac{\partial J_{w}^{T}}{\partial \theta} f$, it can be write:

$$
K_{\theta}=J_{w}^{t} K_{x} J_{m}
$$

Then, the paper will consider :

$$
\delta p=J_{m} K_{\theta}^{-1} J_{w}^{T} W
$$

\section{Flange and wrist center identification}

A serial robot carries a flange on which 3 targets are positionned (Fig. 1). The position of these 3 targets allow to define the position and the orientation of the end effector in the space. A laser tracker with an accuracy of around $0.02 \mathrm{~mm}$ (depending on the distance between the laser tracker and the target) is used. It allows the measurement of the coordinate on a point on the laser tracker frame or in a particular frame which has been identified via the wellknown circle point method[13]. A $R_{0}$ frame located on the robot base is then identified after a revolution of axis 1 and axis 2 . Identification methods permit to localize the targets positioning regarding the modeling of the robot. Then, the circle point method is used to define the link length. The approach leading by [9] considers the barycenter of $P_{10}$, $P_{11}$ and $P_{12}$. To improve this approach, we look for the localization of $P_{\text {wrist }}$, the wrist center i.e. the intersection of the 3 last axis. $P_{T C P}$ is a point on the flange surface which intersects the $6^{\text {th }}$ axis (named : Tool Center Point). These points are defined by $P_{10}, P_{11}$ and $P_{12}$. During the revolution of axis 4 to 6 , they sweep the surface of a sphere whose 
center is $P_{\text {wrist }}$. We present here a metrological method which allows a correct identification of the center of the wrist.

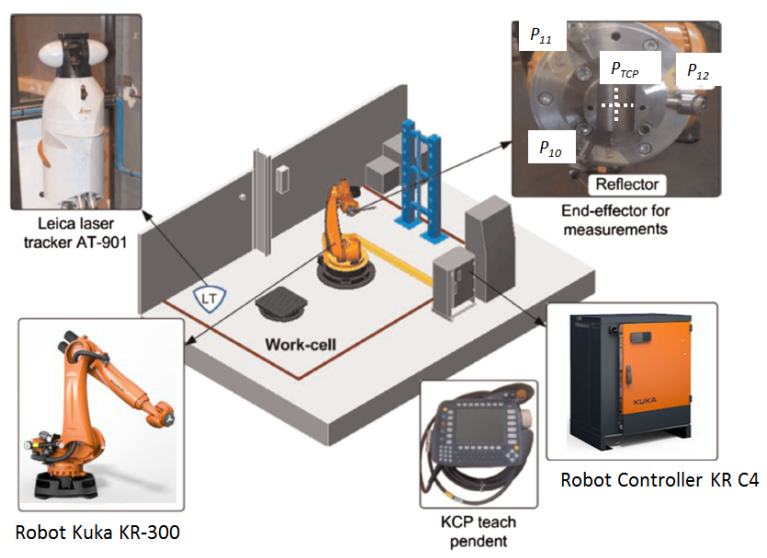

Fig. 1. Our workcell to perform the stiffness identification

Trilateration Algorithm: The knowledge of $P_{\text {wrist }}$ and $P_{T C P}$ is realized in a specific configuration of the robot. In this configuration, $\overrightarrow{x_{0}}$ is colinear to $\overrightarrow{z_{6}}$ (Fig. 2).

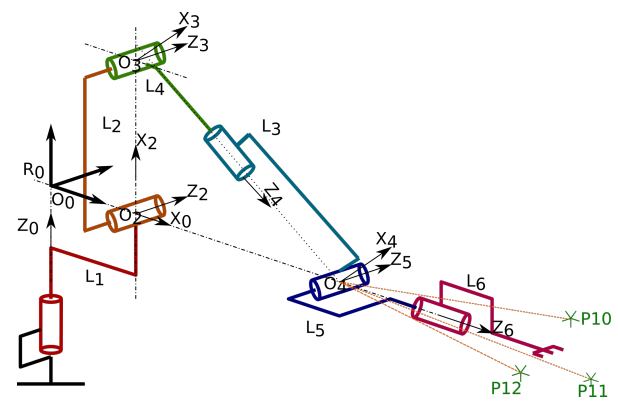

Fig. 2. Construction of the center of the wrist

The use of the circle point method via the revolution of axis 5 and axis 6 allows to define ${ }^{R_{0}} P_{\text {wrist }}$. It is then possible to identify the position of $P_{1 X}$ with $X=0,1,2$ regarding $P_{\text {wrist }}$ via the trilateration formula which identifies $P_{10}, P_{11}$ and $P_{12}$ targets positioning regarding $P_{w r i s t}$ and $P_{T C P}$ (Fig. 3 ). From (13) and (14), we are able to define two wrist centers namely ${ }^{R_{0}} P_{\text {wrist }-1}$ and ${ }^{R_{0}} P_{\text {wrist }-2}$ where the closer of our theoretical one is chosen :

$$
\begin{aligned}
& { }^{R_{0}} P_{\text {wrist }-1}={ }^{R_{0}} P_{1 X}+x e_{x}+y e_{y}+z e_{z} \\
& { }^{R_{0}} P_{\text {wrist }-2}={ }^{R_{0}} P_{1 X}+x e_{x}+y e_{y}-z e_{z}
\end{aligned}
$$

We write here only the formula considering $P_{10}$. The same formula has to be considered with $P_{11}$ and $P_{12}$.

$$
\begin{aligned}
& x=\frac{\left(P_{\text {wrist }} P_{10}\right)^{2}-\left(P_{\text {wrist }} P_{11}\right)^{2}+d^{2}}{2 d} \\
& y=\frac{\left(P_{\text {wrist }} P_{10}\right)^{2}-\left(P_{\text {wrist }} P_{12}\right)^{2}+i^{2}+j^{2}}{2 j}-\frac{i}{j} x \\
& z=\sqrt{\left(P_{\text {wrist }} P_{10}\right)^{2}-x^{2}-y^{2}}
\end{aligned}
$$

and

$$
\begin{array}{lr}
e_{x}=\frac{P_{11}-P_{10}}{\left\|P_{11}-P_{10}\right\|} ; & i=<e_{x}, P_{12}-P_{10}> \\
e_{y}=\frac{P_{12}-P_{10}-<i, e_{x}>}{\left\|P_{12}-P_{10}-<i, e_{x}>\right\|} ; & j=<e_{y}, P_{12}-P_{10}> \\
e_{z}=e_{x} \times e_{y} ; & d=\left\|P_{11}-P_{10}\right\|
\end{array}
$$

These equations allow to normalize the problem. It defines the intersections of 3 spheres. In this way, with 1000 measurements regarding various positions of the $4^{\text {th }}$ to $6^{\text {th }}$ axis, the center of the wrist is defined by less than $0.1 \mathrm{~mm}$ (see Tab. I)

TABLE I

POSITIONNING OFFSET OF THE WRIST

\begin{tabular}{|c||c|c|c|c|}
\hline Name & $X_{\text {mes }}$ & $Y_{\text {mes }}$ & $Z_{\text {mes }}$ & offset \\
\hline$P_{10}$ & 1245.71 & -310.11 & 239.20 & 0.06 \\
\hline$P_{11}$ & 1245.73 & -309.99 & 239.16 & 0.06 \\
\hline$P_{12}$ & 1245.72 & -310.05 & 239.19 & 0.01 \\
\hline Barycenter & 1245.72 & -310.05 & 239.18 & \\
\hline
\end{tabular}

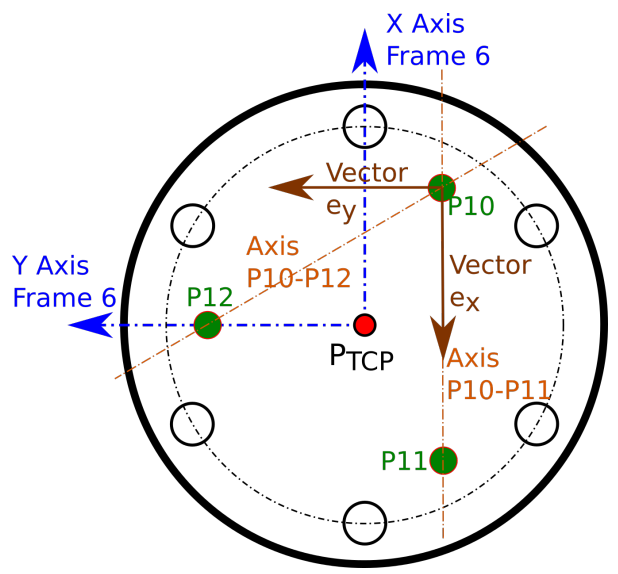

Fig. 3. Definition of the trilateration frame

\section{A NEW METHOD FOR THE STIFFNESS} IDENTIFICATION : DECOUPLING THE ARM AND WRIST

As a novelty regarding the state of the art, we identified the articular stiffness by decoupling the 3 first axis and 3 last one on the industrial robot.

\section{A. Decoupling between the wrist and the arm of the robot}

This expression allows to take into account the location to perform the measurement of the displacement and the force. We consider 2 stiffness matrixes with the stiffness of the 3 first axis $\left(K_{\theta_{123}}\right)$ and the 3 last one $\left(K_{\theta_{456}}\right)$ such as :

$$
K_{\theta}=\left[\begin{array}{cc}
K_{\theta_{123}} & 0 \\
0 & K_{\theta_{456}}
\end{array}\right]
$$




$$
K_{\theta_{123}}=\left[\begin{array}{ccc}
k_{1} & 0 & 0 \\
0 & k_{2} & 0 \\
0 & 0 & k_{3}
\end{array}\right] \text { and } K_{\theta_{456}}=\left[\begin{array}{ccc}
k_{4} & 0 & 0 \\
0 & k_{5} & 0 \\
0 & 0 & k_{6}
\end{array}\right]
$$

Equation (12) becomes :

$$
\delta p=J_{m} K_{\theta_{123}}^{-1} J_{w}^{T} W+J_{m} K_{\theta_{456}}^{-1} J_{w}^{T} W
$$

In order to simplify the lecture of the various equation, we divide the matrixes in 4 sub-matrixes such as:

$$
\begin{array}{r}
J_{m_{i}}=\left[\begin{array}{lll}
J_{m_{i_{1,1}}} & J_{m_{i_{1,2}}} & J_{m_{i_{1,3}}} \\
J_{m_{i_{2,1}}} & J_{m_{i_{2,2}}} & J_{m_{i_{2,3}}} \\
J_{m_{i_{3,1}}} & J_{m_{i_{3,2}}} & J_{m_{i_{3,3}}}
\end{array}\right] \\
J_{w_{i}}=\left[\begin{array}{lll}
J_{w_{i_{1,1}}} & J_{w_{i_{1,2}}} & J_{w_{i_{1,3}}} \\
J_{w_{i_{2,1}}} & J_{w_{i_{2,2}}} & J_{w_{i_{2,3}}} \\
J_{w_{i_{3,1}}} & J_{w_{i_{3,2}}} & J_{w_{i_{3,3}}}
\end{array}\right]
\end{array}
$$

Equation (12) can be write as :

$$
\begin{aligned}
\delta p= & {\left[\begin{array}{ll}
J_{m_{1}} & J_{m_{2}} \\
J_{m_{3}} & J_{m_{4}}
\end{array}\right]\left[\begin{array}{cc}
K_{\theta_{123}}^{-1} & 0 \\
0 & 0
\end{array}\right]\left[\begin{array}{ll}
J_{w_{1}} & J_{w_{2}} \\
J_{w_{3}} & J_{w_{4}}
\end{array}\right]^{T} \cdot W+} \\
& {\left[\begin{array}{ll}
J_{m_{1}} & J_{m_{2}} \\
J_{m_{3}} & J_{m_{4}}
\end{array}\right]\left[\begin{array}{cc}
0 & 0 \\
0 & K_{\theta_{456}}^{-1}
\end{array}\right]\left[\begin{array}{ll}
J_{w_{1}} & J_{w_{2}} \\
J_{w_{3}} & J_{w_{4}}
\end{array}\right]^{T} \cdot W }
\end{aligned}
$$

Using the transposition relation by blocks, the equation becomes :

$$
\begin{aligned}
\delta p= & {\left[\begin{array}{ll}
J_{m_{1}} & J_{m_{2}} \\
J_{m_{3}} & J_{m_{4}}
\end{array}\right]\left[\begin{array}{cc}
K_{\theta_{123}}^{-1} & 0 \\
0 & 0
\end{array}\right]\left[\begin{array}{ll}
J_{w_{1}}^{T} & J_{w_{2}}^{T} \\
J_{w_{3}}^{T} & J_{w_{4}}^{T}
\end{array}\right] \cdot W+} \\
& {\left[\begin{array}{ll}
J_{m_{1}} & J_{m_{2}} \\
J_{m_{3}} & J_{m_{4}}
\end{array}\right]\left[\begin{array}{cc}
0 & 0 \\
0 & K_{\theta_{456}}^{-1}
\end{array}\right]\left[\begin{array}{ll}
J_{w_{1}}^{T} & J_{w_{2}}^{T} \\
J_{w_{3}}^{T} & J_{w_{4}}^{T}
\end{array}\right] \cdot W } \\
\delta p= & {\left[\begin{array}{ll}
J_{m_{1}} & J_{m_{2}} \\
J_{m_{3}} & J_{m_{4}}
\end{array}\right]\left[\begin{array}{cc}
K_{\theta_{123}}^{-1} J_{w_{1}}^{T} & K_{\theta_{123}}^{-1} J_{w_{3}}^{T} \\
0 & 0
\end{array}\right] \cdot W+} \\
& {\left[\begin{array}{ll}
J_{m_{1}} & J_{m_{2}} \\
J_{m_{3}} & J_{m_{4}}
\end{array}\right]\left[\begin{array}{cc}
0 & 0 \\
K_{\theta_{456}}^{-1} \cdot J_{w_{2}}^{T} & K_{\theta_{456}}^{-1} \cdot J_{w_{4}}^{T}
\end{array}\right] \cdot W }
\end{aligned}
$$

We can finally write $\delta p$ following a matricial product linked to the arm stiffness and a matricial product linked to the wrist stiffness :

$$
\begin{aligned}
\delta p= & {\left[\begin{array}{ll}
J_{m_{1}} \cdot K_{\theta_{123}}^{-1} \cdot J_{w_{1}}^{T} & J_{m_{1}} \cdot K_{\theta_{12}}^{-1} \cdot J_{w_{3}}^{T} \\
J_{m_{3}} \cdot K_{\theta_{123}}^{-1} \cdot J_{w_{1}}^{T} & J_{m_{3}} \cdot K_{\theta_{123}}^{-1} \cdot J_{w_{3}}^{T}
\end{array}\right] \cdot W+} \\
& {\left[\begin{array}{ll}
J_{m_{2}} \cdot K_{\theta_{45}}^{-1} \cdot J_{w_{2}}^{T} & J_{m_{2}} \cdot K_{\theta_{456}}^{-1} \cdot J_{w_{4}}^{T} \\
J_{m_{4}} \cdot K_{\theta_{456}}^{-1} \cdot J_{w_{2}}^{T} & J_{m_{4}} \cdot K_{\theta_{456}}^{-1} \cdot J_{w_{4}}^{T}
\end{array}\right] \cdot W }
\end{aligned}
$$

We introduce now the values of the wrench $W$ :

$$
W=\left[\begin{array}{ll}
F & M
\end{array}\right]^{T}=\left[\begin{array}{llllll}
F_{x} & F_{y} & F_{z} & M_{x} & M_{y} & M_{z}
\end{array}\right]^{T}
$$

In order to perform the identification, we only consider the position of the end effector i.e. we will use only $\delta p_{m}$. Moreover, considering only the arm about (23) (Only $K_{\theta_{123}}^{-1}$ ), the first part of (29) can be developed and reduced. We lead to this equation :

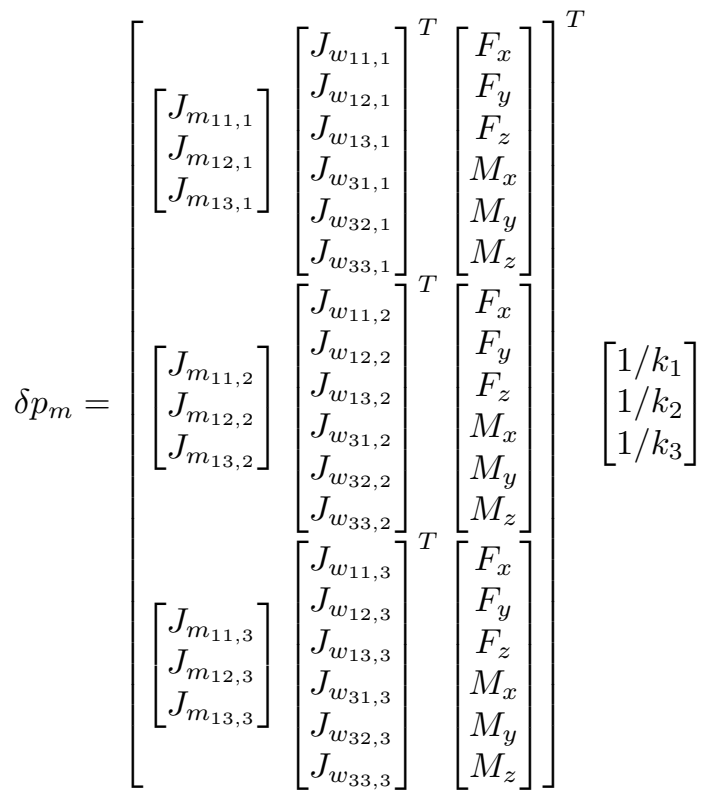

In order to solve the problem, it is necessary to condition the equations in order to be able to construct $A_{\text {arm }}$ and $A_{\text {wrist }}$. In order to avoid a geometrical transformation, $A_{\text {wrist }}^{P_{1 X}}$ is considered on a given point $P_{1 X}$ with $\mathrm{X}=0,1,2$ such as:

$$
\begin{aligned}
& A_{\text {arm }} k_{\text {arm }}=\delta d_{\text {arm }} \quad \text { with } \quad k_{\text {arm }}=\left[\begin{array}{lll}
\frac{1}{k_{\theta_{1}}} & \frac{1}{k_{\theta_{2}}} & \frac{1}{k_{\theta_{3}}}
\end{array}\right]^{T} \\
& A_{\text {wrist }}^{P_{1 X}} k_{\text {wrist }}=\delta d_{\text {wrist }}^{P_{1 X} \text { with }} k_{\text {wrist }}=\left[\begin{array}{lll}
\frac{1}{k_{\theta_{4}}} & \frac{1}{k_{\theta_{5}}} & \frac{1}{k_{\theta_{6}}}
\end{array}\right]^{T}
\end{aligned}
$$

In order to evaluate the arm stiffness, we define two matrixes $B_{\text {arm }}$ and $C_{\text {arm }}$ such as :

$$
B_{\text {arm }}=\left[\begin{array}{lllll}
A_{\text {arm-1 }}^{1} & \ldots & A_{\text {arm-i }}^{j} & \ldots & A_{\text {arm-m }}^{p}
\end{array}\right]_{\substack{i=1, \ldots, m \\
j=1, \ldots, p}}^{T}
$$

$C_{\text {arm }}=\left[\begin{array}{lllll}\delta d_{\text {arm }-1} & \ldots & \delta d_{\text {arm }-i} & \ldots & \delta d_{\text {arm }-m}\end{array}\right]_{i=1,}^{T}$.

$p$ is the number of measurement points and $m$ is the number of tested configuration. In this way, the state vector $\left[\begin{array}{lll}1 / k_{1} & 1 / k_{2} & 1 / k_{3}\end{array}\right]^{T}$ is able to converge to $\left[\begin{array}{lll}1 / k_{\theta_{1}} & 1 / k_{\theta_{2}} & 1 / k_{\theta_{3}}\end{array}\right]^{T}$. In order to identify the wrist stiffness, we followed the same procedure :

$$
\delta p_{\text {wrist }}^{P_{1 X}}=A_{\text {wrist }}^{P_{1 X}} k_{w r i s t}
$$

and

$$
\delta p_{\text {wrist } 1 X}^{P}=\delta p_{\text {tot }}^{P_{1 X}}-\delta p_{\text {wrist-model }}^{1 X}
$$

with 
$\delta p_{\text {wrist-model }}^{1 X}=\left[\begin{array}{ll}J_{m_{1}} & J_{m_{2}} \\ J_{m_{3}} & J_{m_{4}}\end{array}\right]\left[\begin{array}{cc}K_{\theta_{123}}^{-1} & 0 \\ 0 & 0\end{array}\right]\left[\begin{array}{cc}J_{w_{1}} & J_{w_{2}} \\ J_{w_{3}} & J_{w_{4}}\end{array}\right] . W$

Where $J_{m}$ and $J_{w}$ are written at the measured point $P_{1 X}$ with $\mathrm{X}=0,1,2$.

\section{APPLICATION ON AN INDUSTRIAL ROBOT WITH DOUBLE ENCODING SYSTEM}

\section{A. Complete pose Method (Fig. 4)}

We develop the approach on an industrial robot with double encoding system to identify our new approach to identify the articular stiffness. We compare the three approaches : 2 were defined in the state of the art. It concerns the Complete Pose[9] based on the reconstruction of a frame (center $\left.P_{T C P}\right)$ on the flange based on the measurement of $P_{10}$ to $P_{12}$ in $R_{0}$. The calculation of the loading $W$ is positioned at $P_{T C P}$ with a geometrical transformation. The Jacobian ${ }^{R_{0}} J_{P_{T C P}}$ is defined from the geometrical model with the link length calculed via the circle point method. The definition of the articular stiffness uses the following equation which takes into account the positioning, the rotation of the flange and the normalization of the Jacobian matrix.

$$
\delta p={ }^{R_{0}} J_{N_{P_{T C P}}} K_{\theta}{ }^{R_{0}} J_{N_{P_{T C P}}^{T}}^{T} W_{P_{T C P}}
$$

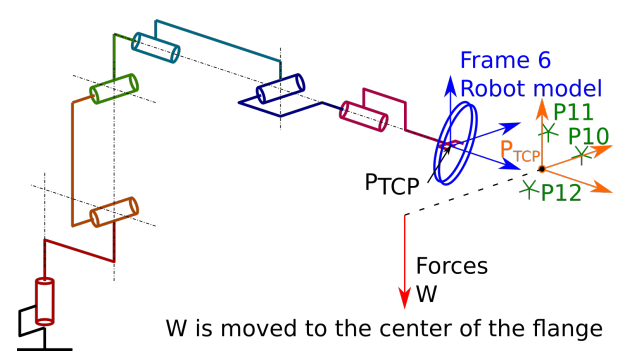

Fig. 4. Approach followed by [2]

\section{B. Partial Pose method (Fig. 5)}

The partial pose method proposed by[10] uses a numerical optimization of the robot modelling and tools transformation to link $P_{10}$ to $P_{12}$ to the flange. This method uses direct measurement data only. This approach aimed at finding the configuration that ensures the best robot positioning accuracy after geometric error compensation. The measured loading $W_{\text {measured }}$ allows to define the articular stiffness with a Jacobian in a consistent way via numerical optimization. The following equation is used considering only the positioning $\delta p_{m}$.

$$
\delta p_{m}={ }^{R_{0}} J_{P_{1 X}} K_{\theta}{ }^{R_{0}} J_{w}^{T} W_{\text {measured }}
$$

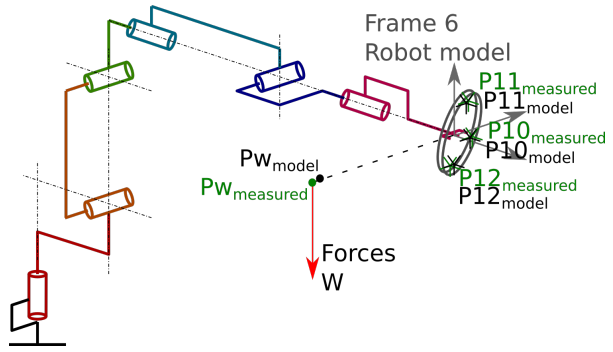

Fig. 5. Approach followed by [5]

\section{Decoupled Partial Pose method (Fig. 6)}

In our approach, we take the best of the two methods presented before. The measurement of $P_{10}$ to $P_{12}$ is performed in $R_{0}$ and no geometrical transformation is applied. To use the decoupling effect, the loading is placed at the center of the wrist via the trilateration method which allows a metrological knowledge of the manipulator. In this way, 4 Jacobian matrixes are defined such as ${ }^{R_{0}} J_{P_{10}},{ }^{R} J_{P_{11}}$, ${ }^{R} J_{P_{12}}$ and ${ }^{R_{0}} J_{P_{\text {wrist }}}$.

$$
\delta p_{m}={ }^{R_{0}} J_{P_{1 X}} K_{\theta}{ }^{R_{0}} J_{w}^{T} W_{P_{w r i s t}} \text { with } \quad X=0,1,2
$$

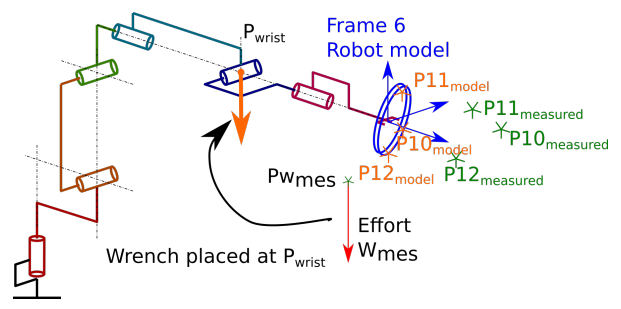

Fig. 6. Our new approach

\section{Comparison between the three methods}

TABLE II

\begin{tabular}{|c|c|c|c|c|c|}
\hline Method & $k_{\theta_{2}}$ & $k_{\theta_{3}}$ & $k_{\theta_{4}}$ & $k_{\theta_{5}}$ & $k_{\theta_{6}}$ \\
\hline $\begin{array}{l}\mathrm{CP} \text { inac- } \\
\text { tive }\end{array}$ & $\begin{array}{l}3.2 \& \\
0.58\end{array}$ & $\begin{array}{l}1.5 \\
0.43\end{array}$ & $\begin{array}{ll}0.24 \& \\
2.7\end{array}$ & $\begin{array}{l}0.21 \& \\
0.026\end{array}$ & $\begin{array}{l}0.051 \& \\
0.012\end{array}$ \\
\hline $\begin{array}{l}\mathrm{CP} \\
\text { active }\end{array}$ & $\begin{array}{ll}7.4 & \& \\
8.5 & \end{array}$ & $\begin{array}{ll}2.1 & \& \\
1.7 & \end{array}$ & $\begin{array}{l}-0.24 \& \\
0.22\end{array}$ & $\begin{array}{l}0.37 \& \\
0.12\end{array}$ & $\begin{array}{l}0.44 \& \\
0.007\end{array}$ \\
\hline $\begin{array}{l}\mathrm{PP} \text { inac } \\
\text { tive }\end{array}$ & $\begin{array}{l}3.4 \quad \& \\
0.067\end{array}$ & $\begin{array}{l}2.2 \& \\
0.06\end{array}$ & $\begin{array}{l}0.89 \& \\
0.21\end{array}$ & $\begin{array}{ll}6.9 & \& \\
22 & \end{array}$ & $\begin{array}{l}0.11 \& \\
0.028\end{array}$ \\
\hline $\begin{array}{l}\mathrm{PP} \\
\text { active }\end{array}$ & $\begin{array}{l}6.5 \& \\
0.27\end{array}$ & $\begin{array}{l}4 \quad \& \\
0.25\end{array}$ & $1 \& 0.3$ & $\begin{array}{l}2.7 \& \\
2300\end{array}$ & $\begin{array}{l}0.087 \& \\
0.014\end{array}$ \\
\hline $\begin{array}{l}\text { DPP in- } \\
\text { active }\end{array}$ & $\begin{array}{l}3.2 \& \\
0.039\end{array}$ & $\begin{array}{l}2.6 \& \text { \& } \\
0.04\end{array}$ & $\begin{array}{l}0.42 \& \& \\
0.057\end{array}$ & $\begin{array}{l}0.23 \& \\
0.008\end{array}$ & $\begin{array}{l}0.089 \& \\
0.018\end{array}$ \\
\hline $\begin{array}{l}\text { DPP ac- } \\
\text { tive }\end{array}$ & $\begin{array}{ll}5.6 & \& \\
0.1 & \end{array}$ & $\begin{array}{l}6.3 \& \\
0.22\end{array}$ & $\begin{array}{l}0.46 \& \text { \& } \\
0.057\end{array}$ & $\begin{array}{l}0.35 \& \\
0.012\end{array}$ & $\begin{array}{l}0.079 \& \\
0.012\end{array}$ \\
\hline
\end{tabular}

IDENTIFICATION METHODS USED TO IDENTIFY THE ARTICULAR STIFFNESS : MEAN \& STANDARD DEVIATION $-10^{6} \mathrm{Nm} / \mathrm{rad}$

To highlight the performances of our new method, we active the double encoding system. It leads to a stiffer arm which allows to compare the different approaches. We present in Tab. II, the values of the articular stiffness (mean and standard deviation). We highlight in "bold" the values 
which not to seem correct on our point of view. With the Complete Pose method, we notice that the stiffness of the $4^{\text {th }}$ axis is under-estimated and can be negative when activating the double encoding system. As far as Partial Pose without decoupling is concerned, the $5^{t h}$ axis is not given with an enough accuracy based on the standard deviation. We present now the cartesian deflection to evaluate the quality of the convergence of our algorithm.

\section{E. Cartesian deflection}

Cartesian deflection shows the impact of a loading on the stiffness behavior of the robot. Based on this asumption, we notice, because of the anisotropic behavior of the robot, that for the same loading, the displacement of $P_{\text {wrist }}$ is not the same. This value is the norm of $\delta p_{m}$. For the same loading, the activation of the double encoding system allows to avoid $40 \%$ of the deflection with double encoding disabled (considered as a reference to be compensated). The deflection with the activation of the double encoding system is shown in grey. To evaluate the performance of our algorithm, we consider 19 configurations with double encoding enabled. Randomly, on 10 configurations, we evaluate the articular stiffness and we apply it on the 19 configurations. With the CP method (Complete Pose method), we obtain a high variability and we are able to compensate $50 \%$ of the deflection. With the PP method (Partial Pose method), the compensation is better $(80 \%)$ and with the DPP method (Decoupling Partial Pose method) we reach a better compensation (84\%). In this way, we present at Fig. 7, the deflection and we make a focus more specifically on the PP and DPP method (Fig. 8).

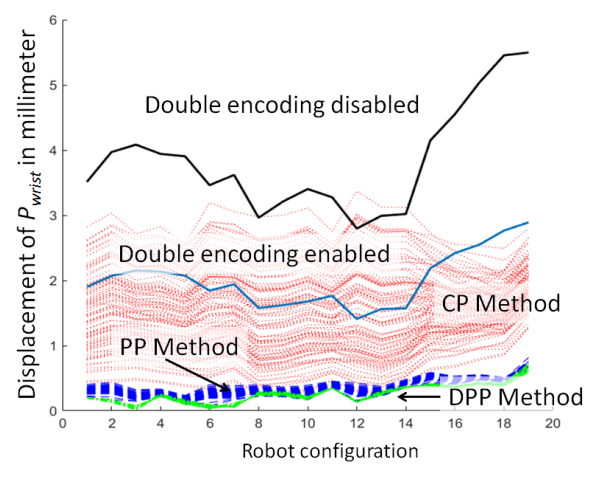

Fig. 7. Robot deflection : global deflection (grey), after compensation with CP method (red), after compensation with PP method (blue), with DPP method (green)

\section{CONCLUSION AND PERSPECTIVES}

In this paper, we present a new indentification method for a better evaluation of the articular stiffness of an industrial robot. We based our approach on a metrological measurement with a physical meaning. We propose a geometrical definition of the flange transformation. We bring in this way a better localization of the center of the wrist via the trilateration method. This allows to remove a variability on the loading calculation to take it into account at the center of the wrist. The results highlight that we are able to take

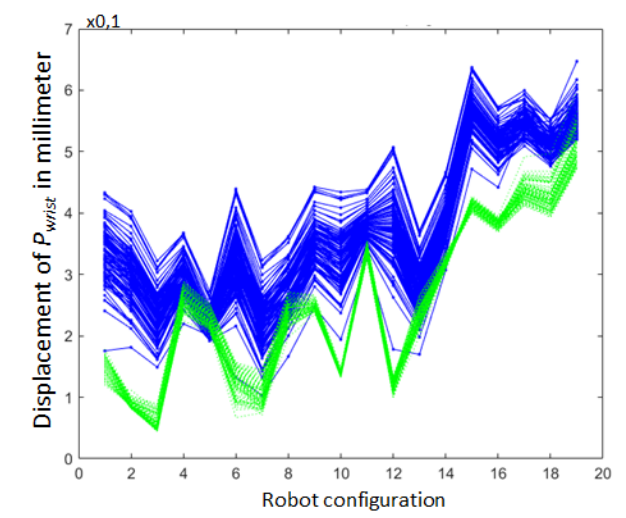

Fig. 8. Zoom based on the previous picture : PP method (blue) and DPP one (green)

into account $84 \%$ of the deflection. Unfortunately, we do not have a perfect solution to compensate the positioning of the robot in its workspace. To improve it, we can first look for better postures to perform the identification. Secondly, the modelling take into account the behavior of the joint. Links stiffness must be considered. In all the case, the process must be studied to adapt the quality of the stiffness identification.

\section{REFERENCES}

[1] S.-F. Chen and I. Kao, "Geometrical approach to the conservative congruence transformation (cct) for robotic stiffness control," vol. 1, pp. 544-549, 2002.

[2] C. Dumas, S. Caro, S. Garnier, and B. Furet, "Workpiece placement optimization of six-revolute industrial serial robots for machining operations," in ASME 2012 11th Biennial Conference on Engineering Systems Design and Analysis, pp. 419-428, American Society of Mechanical Engineers, 2012.

[3] K. Subrin, L. Sabourin, R. Cousturier, G. Gogu, and Y. Mezouar, "New redundant architectures in machining: serial and parallel robots," Procedia Engineering, vol. 63, pp. 158-166, 2013.

[4] M. Cherif, J.-Y. Knevez, and A. Ballu, "Thermal aspects on robot machining accuracy," Proceedings of IDMME-Virtual Concep, vol. 48, 2010.

[5] A. Nubiola and I. A. Bonev, "Absolute calibration of an abb irb 1600 robot using a laser tracker," Robotics and Computer-Integrated Manufacturing, vol. 29, no. 1, pp. 236-245, 2013.

[6] A. Nubiola and I. A. Bonev, "Absolute robot calibration with a single telescoping ballbar," Precision Engineering, vol. 38, no. 3, pp. 472480, 2014.

[7] G. Du and P. Zhang, "Online robot calibration based on vision measurement," Robotics and Computer-Integrated Manufacturing, vol. 29, no. 6, pp. 484-492, 2013.

[8] F. Leali, A. Vergnano, F. Pini, M. Pellicciari, and G. Berselli, "A workcell calibration method for enhancing accuracy in robot machining of aerospace parts," The International Journal of Advanced Manufacturing Technology, vol. 85, no. 1, pp. 47-55, 2016.

[9] C. Dumas, S. Caro, S. Garnier, and B. Furet, "Joint stiffness identification of six-revolute industrial serial robots," Robotics and ComputerIntegrated Manufacturing, vol. 27, no. 4, pp. 881-888, 2011.

[10] Y. Wu, A. Klimchik, S. Caro, B. Furet, and A. Pashkevich, "Geometric calibration of industrial robots using enhanced partial pose measurements and design of experiments," Robotics and Computer-Integrated Manufacturing, vol. 35, pp. 151-168, 2015.

[11] W. A. Khan and J. Angeles, "The kinetostatic optimization of robotic manipulators: the inverse and the direct problems," Journal of mechanical design, vol. 128, no. 1, pp. 168-178, 2006.

[12] M. R. Driels and W. E. Swayze, "Automated partial pose measurement system for manipulator calibration experiments," IEEE Transactions on Robotics and Automation, vol. 10, no. 4, pp. 430-440, 1994.

[13] B. W. Mooring, Z. S. Roth, and M. R. Driels, Fundamentals of manipulator calibration. Wiley New York, 1991. 